九州大学学術情報リポジトリ

Kyushu University Institutional Repository

\title{
Pseudo Extension in Default Reasoning and Belief Revision by Model Inference
}

Yuasa, Hiroko

Department of Information Systems, Kyushu University

Arikawa, Setsuo

Research Institute of Fundamental Information Science Kyushu University

http://hdl. handle. net/2324/3110

出版情報: RIFIS Technical Report. 5, 1988-10-22. Research Institute of Fundamental Information Science, Kyushu University

バージョン:

権利関係 : 


\title{
RIFIS Technical Report
}

\author{
Pseudo Extension in Default Reasoning \\ and \\ Belief Revision by Model Inference
}

\author{
Hiroko Yuasa \\ Setsuo Arikawa
}

October 22, 1988

Research Institute of Fundamental Information Science

Kyushu University 33

Fukuoka 812, Japan 


\title{
Pseudo Extension in Default Reasoning and
} Belief Revision by Model Inference

\author{
Hiroko Yuasa* and Setsuo Arikawa \\ Department of Information Systems, \\ Kyushu University 39, Kasuga 816, Japan \\ Mailing address: S. Arikawa, Research Institute of Fundamental Information Science, \\ Kyushu University 33, Fukuoka 812, Japan
}

\begin{abstract}
This paper is concerned with extension and belief revision in Reiter's default reasoning. First we introduce a new notion of pseudo extension which is successively constructed from a given default theory. We show that the set of pseudo extensions contains the set of extensions, so that we can get some reasonable candidates of extension in a constructive way. This should be a first step towards computerization of the default reasoning. We also discuss the belief revision problem from the viewpoint of Shapiro's inductive inference of models.
\end{abstract}

\section{Introduction}

Reasoning we use in our daily problem solving is not always from complete information unlike mathematical reasoning. One of the main goals of artificial intelligence research is to computerize our reasoning. Hence much attention has been paid to such reasoning and formulations have been proposed by many authors. Reiter's default reasoning is one such formulation. He used the notion of default and formulated incomplete knowledge about a world as a pair : a set of true but incomplete knowledge and a set of special inference rules called defaults. He enlarged the incomplete knowledge to describe the world as completely

* Presently at Hitachi Central Research Laboratory, Hitachi Ltd. 
as possible. The enlarged knowledge is called an extension, which is the set of knowledge that seems true as a result of reasoning from incomplete knowledge and is a possible world at that time. Thus the extension is not definite knowledge but just a belief about the world, and hence it needs to be modified when new facts are found.

In the Reiter's default theory we can reason from incomplete knowledge in a very natural way. However, the extension can not successively be constructed, because it is defined by using itself in applying the defaults.

In the present paper, we first try to solve this difficulty. We define a successively constructable set of knowledge, which we call a pseudo extension, and discuss the relationship between Reiter's extensions and ours. We also show that our extension is a natural generalization of Reiter's.

Unlike the reasoning in classical logic, the reasoning from incomplete knowledge is necessarily non-monotonic. Hence we sometimes must modify the belief, i.e. the knowledge derived by such reasoning. In the present paper we take this problem of belief revision as that of extension modification. Then we can see that the process of belief revision is very similar to Shapiro's model inference. We also discuss the belief revision from this viewpoint.

\section{Reiter's Default Reasoning}

We start with recalling the definitions and important results on default reasoning according to $[1,2]$.

Let $L$ be a first order language. Then a default is a rule of the form

$$
\frac{\alpha(x): \mathbf{M} \beta_{1}(x), \ldots, \mathbf{M} \beta_{m}(x)}{\omega(x)},
$$

where $\alpha(x), \beta_{1}(x), \cdots, \beta_{m}(x), \omega(x)$ are well-formed formulas (wff, for short) in $L$, and $x$ denotes a sequence of free variables. We call $\alpha(x)$ a prerequisite, $\beta_{1}(x), \ldots, \beta_{m}(x)$ justifications, and $\omega(x)$ a consequence.

The $\mathbf{M}$ is a special symbol to be read as "it is consistent to assume". Hence the default means that if it is consistent to assume $\beta_{1}(x), \ldots, \beta_{m}(x)$ we may infer $\alpha(x) \rightarrow \omega(x)$. Note that "it is consistent to assume $\beta_{i}(x)$ " means that there is not sufficient information to 
conclude $\neg \beta_{i}(x)$. The prerequisite $\alpha(x)$ may possibly be empty, that is,

$$
\frac{: \mathbf{M} \beta_{1}(x), \ldots, \mathbf{M} \beta_{m}(x)}{\omega(x)}
$$

is also a default to mean that if it is consistent to assume $\beta_{1}(x), \ldots, \beta_{m}(x)$ then $\omega(x)$ can be concluded.

If $\alpha(x), \beta_{1}(x), \ldots, \beta_{m}(x), \omega(x)$ have no free variables, the rule is called a closed default and denoted

$$
\frac{\alpha: \mathbf{M} \beta_{1}, \ldots, \mathbf{M} \beta_{m}}{\omega}
$$

A default of the form

$$
\frac{\alpha(x): \mathbf{M} \beta(x)}{\beta(x)}
$$

is called a normal default.

Now let $D$ be a set of defaults, and $W$ be a set of closed wffs. Then $\Delta=(D, W)$ is called a default theory. A default theory $\Delta$ is closed if every default in $D$ is closed, and $\Delta$ is normal if every default in $D$ is normal.

For a set $D$ of default rules, we define $C o n s(D)$ by

$$
\operatorname{Cons}(D)=\left\{\omega(x) \mid \frac{\alpha(x): \mathbf{M} \beta_{1}(x), \ldots, \mathbf{M} \beta_{m}(x)}{\omega(x)} \in D\right\}
$$

Thus $\operatorname{Cons}(D)$ is the set of all consequences of defaults in $D$.

For a set $S$ of closed wffs and a closed wff $\omega$, we write $S \vdash \omega$ to denote that $\omega$ is derived from $S$, and $S \nvdash \omega$ to denote that $\omega$ is not derived from $S$. We also define a set $T h(S)$ by

$$
T h(S)=\{\omega \mid \omega \text { is a closed wff and } S \vdash \omega\}
$$

Let $\Delta=(D, W)$ be a closed default theory, $S \subseteq L$ be a set of closed wffs, and $\Gamma(S)$ be the smallest set satisfying the following three properties:

(1) $W \subseteq \Gamma(S)$.

(2) $\operatorname{Th}(\Gamma(S))=\Gamma(S)$.

(3) If $\frac{\alpha: \mathbf{M} \beta_{1}, \ldots, \mathbf{M} \beta_{m}}{\omega} \in D, \alpha \in \Gamma(S), \neg \beta_{1}, \ldots, \neg \beta_{m} \notin S$ then $\omega \in \Gamma(S)$.

A set $E \subseteq L$ satisfying $\Gamma(E)=E$ is an extension of $\Delta$. An intuitive characterization of the extensions is given by the following theorem : 
Theorem 1 (Reiter [1]) Let $E \subseteq L$ be a set of closed wffs, $\Delta=(D, W)$ be a closed default theory, and $E_{0}, E_{1}, \ldots$ be the following sequence of sets of closed wffs:

$$
\begin{aligned}
& E_{0}=W
\end{aligned}
$$

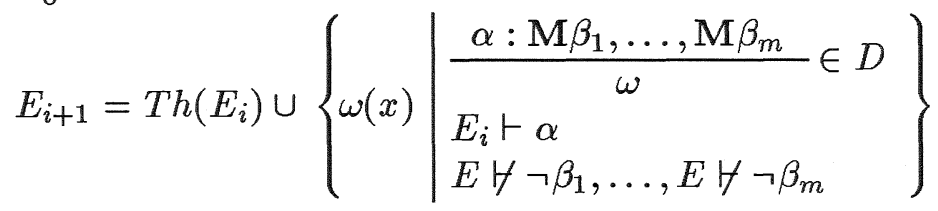

Then $E$ is an extension of $\Delta$ if and only if

$$
E=\bigcup_{i=0}^{\infty} E i
$$

The expression of $E_{i+1}$ in Theorem 1 is slightly different from the original one. Reiter [1] used conditions $\alpha \in E_{1}, \neg \beta_{1}, \ldots, \neg \beta_{m} \notin E$ instead of $E_{i} \vdash \alpha, E \nvdash \neg \beta_{1}, \ldots, E \nvdash$ $\neg \beta_{m}$, respectively. These expressions are essentially the same. In order to check $\alpha \in$ $E_{1}, \neg \beta_{1}, \ldots, \neg \beta_{m} \in E$, we need to check whether they are derivable or not. So we have adopted our expression, which should be more intuitive.

For a closed default theory there may exist two or more extensions or none at all. The existence of many extensions means the possibility of many worlds at that time. Selecting a correct extension and constructing an extension are different things. The correctness will gradually become clear as new facts about the world are known. We say that a closed default theory has an inconsistent extension if one of its extensions is the set of all closed wffs of $L$.

Corollary 2 (Reiter [1]) A closed default theory $(D, W)$ has an inconsistent extension if and only if $W$ is inconsistent.

Corollary 3 (Reiter [1]) If a closed default theory $\Delta$ has an inconsistent extension then it is only the extension of $\Delta$.

Since a closed default theory with an inconsistent $W$ has only inconsistent extensions, hereafter we restrict our discussions to default theories with consistent $W$ 's, unless otherwise stated. Default theory may not have extensions, but we know the following :

Theorem 4(Reiter [1]) Every closed normal default theory has at least one extension. 


\section{Pseudo Extensions and Their Properties}

In defining extension $E$, Reiter used $E$ itself (Theorem 1). Hence it is a self-recursive definition. We can use Theorem 1 just to confirm for a given set of closed wffs to be an extension, but can not use it to construct extensions from a given closed default theory.

This section proposes a new kind of extensions which can successively be constructed from a closed default theory.

Definition Let $\Delta=(D, W)$ be a closed default theory and let $F_{i}(i \geq 0)$ be sets of closed wffs defined as follows:

$$
\begin{aligned}
& F_{0}=W \\
& F_{i+1}=T h\left(F_{i}\right) \cup \operatorname{Cons}\left(D_{F_{i}}\right) \quad(i \geq 0),
\end{aligned}
$$

where $D_{F_{i}}$ is a subset of

$$
\left\{\begin{array}{l|l}
\delta=\frac{\alpha: \mathbf{M} \beta_{1}, \ldots, \mathbf{M} \beta_{m}}{\omega} & \begin{array}{l}
\delta \in D, \omega \notin \operatorname{Cons}\left(\bigcup_{k=1}^{i-1} D_{F_{k}}\right) \\
F_{i} \vdash \alpha \\
F_{i} \nvdash \neg \beta_{1}, \ldots, F_{i} \nvdash \neg \beta_{m}
\end{array}
\end{array}\right\}
$$

such that $D_{F_{i}} \neq \emptyset$ if the above set is not empty. Then we call

$$
F=\bigcup_{i=0}^{\infty} F_{i}
$$

a pseudo extension of the theory $\Delta$.

The condition $F_{i} \forall \neg \beta_{1}, \ldots, F_{i} \not \forall \beta_{m}$ in applying a default with $\mathbf{M} \beta_{1}, \ldots, \mathbf{M} \beta_{m}$ is diffrent from Reiter's. Now we can successively construct the pseudo extensions. Moreover in constructing $F_{i+1}$ we can use an appropriate subset of defaults instead of the whole. A relation between Reiter's extension and ours is given as follows :

Theorem 5 Let $\tilde{E}$ be the set of all extensions of a closed default theory $\Delta$, and let $\tilde{F}$ be the set of all pseudo extensions of $\Delta$. Then

$$
\tilde{E} \subseteq \tilde{F}
$$

Therefore every extension is also a pseudo extension. 
Proof Let $E=\bigcup_{i=1}^{\infty} E_{i}$ be any extension, and $D_{i}$ be the set of defaults used in defining $E_{i}$. Then we have

$$
E_{i}=T h\left(E_{i-1}\right) \cup \operatorname{Cons}\left(D_{i}\right)
$$

For a default

$$
\delta=\frac{\alpha: \mathbf{M} \beta_{1}, \ldots, \mathbf{M} \beta_{m}}{\omega} \in D_{i}
$$

we easily have

$$
\begin{aligned}
& E_{i} \vdash \alpha \\
& E \nvdash \neg \beta_{1}, \ldots, E \forall \neg \beta m .
\end{aligned}
$$

By putting

$$
D_{i}^{\prime}=\left\{\delta \mid \delta \in D_{i}, \operatorname{Cons}(\delta) \notin F_{i-1}\right\},
$$

we construct sets of closed wffs $F_{1}, F_{2}, \ldots$ as follows :

$$
\begin{aligned}
& F_{0}=W \\
& F_{1}=T h\left(F_{0}\right) \cup \operatorname{Cons}\left(D_{1}^{\prime}\right) \\
& \quad \vdots \\
& F_{i}=T h\left(F_{i-1}\right) \cup \operatorname{Cons}\left(D_{i}^{\prime}\right)
\end{aligned}
$$

Then, since $E_{i}=F_{i}$ for each $i(\geq 0)$, we have

$$
E=\bigcup_{i=0}^{\infty} E_{i}=\bigcup_{i=1}^{\infty} F_{i} .
$$

Now let us show that $D_{i}^{\prime}$ satisfies the condition of $D_{F_{i}}$ in the definition of pseudo extensions. First assume that for some $j$ and for any $k(\geq j)$ a default satisfies the condition but $D_{k}^{\prime}=\emptyset$. That is, assume that the set

$$
\left\{\begin{array}{l|l}
\delta=\frac{\alpha: \mathbf{M} \beta_{1}, \ldots, \mathbf{M} \beta_{m}}{\omega} & \begin{array}{l}
\delta \in D, \omega \notin \operatorname{Cons}\left(\bigcup_{k=1}^{i-1} D_{k}^{\prime}\right) \\
F_{i} \vdash \alpha \\
F_{i} \nvdash \neg \beta_{1}, \ldots, F_{i} \forall \neg \beta_{m}
\end{array}
\end{array}\right\}
$$

is not empty but $D_{k}^{\prime}$ is empty. Then by definition of $D_{i}^{\prime}$ and $D_{k}^{\prime}=\emptyset$, we have

$$
D_{j-1}=D_{j}=D_{j+1}=\ldots
$$


Hence we have

$$
E_{j}=E_{j+1}=E_{j+2}=\ldots
$$

From the assumption there exists a default

$$
\delta=\frac{\alpha: \mathbf{M} \beta_{1}, \ldots, \mathbf{M} \beta_{m}}{\omega} \in D,
$$

which satisfies

$$
\begin{aligned}
& \omega \notin \operatorname{Cons}\left(\bigcup_{k=1}^{j-1} D_{k}^{\prime}\right) \\
& F_{j} \vdash \alpha \\
& F_{j} \forall \neg \beta_{1}, \ldots, F_{j} \forall \neg \beta_{m} .
\end{aligned}
$$

By $E_{j}=F_{j}$, obviously

$$
\begin{aligned}
& E_{j} \vdash \alpha \\
& E_{j} \nvdash \neg \beta_{1}, \ldots, E_{j} \nvdash \neg \beta_{m} .
\end{aligned}
$$

Also by

$$
\omega \notin \operatorname{Cons}\left(\bigcup_{i=1}^{\infty} D_{i}^{\prime}\right) \subseteq \operatorname{Cons}\left(\bigcup_{i=1}^{\infty} D_{i}\right),
$$

there exists at least one $k(1 \leq k \leq m)$ such that

$$
E \vdash \neg \beta_{k}
$$

Since $E=\bigcup_{i=0}^{\infty} E_{i}$, there exists an $i$ such that $E_{i} \vdash \neg \beta_{k}$. This $i$ must be $i>j$, because $E_{i} \forall \neg \neg \beta_{k}$ for $i \leq j$. Since $E_{i}=E_{j}$ for such $i, j$, we have

$$
E_{j} \vdash \neg \beta_{k}
$$

Also by $E_{j}=F_{j}$, we have

$$
F_{j} \vdash \neg \beta_{k},
$$

which contradicts

$$
F_{j} \forall \neg \beta_{1}, \ldots, F_{j} \forall \neg \beta_{m} \text {. }
$$

Therefore $D_{i}^{\prime}$ satisfies the condition of $D_{F_{i}}$, and hence $F=\bigcup_{i=0}^{\infty} F_{i}$ is a pseudo extension. Thus we have

$$
E=\bigcup_{i=0}^{\infty} E_{i}=\bigcup_{i=0}^{\infty} F_{i}=F \in \tilde{F}
$$

Hence $\tilde{E} \subseteq \tilde{F}$. 
Theorem 6 In a closed normal default theory, any pseudo extension which is not an extension is inconsistent.

Proof Let $F$ be a set of closed wffs such that $F \in \tilde{F}$ and $F \notin \tilde{E}$. Let $D_{F_{i}}$ be the set of defaults which is used in constructing $F_{i}$ of $F=\bigcup_{i=0}^{\infty} F_{i}$. Then by the assumption there exist an i and a default

$$
\delta=\frac{\alpha: \mathbf{M} \beta}{\beta}
$$

such that $\delta \in D_{F_{i}}, F \vdash \alpha, F_{i} \nvdash \neg \beta, F \vdash \beta$. By $\delta \in D, F \vdash \alpha, F_{i} \forall \neg \neg \beta$, we have

$$
\beta \in F_{i+1} \subseteq F
$$

Hence $F \vdash \neg \beta$ and $F \vdash \beta$, which means $F$ is inconsistent.

Remark Theorem 6 is not valid if the default theory is not normal. In fact, there exists a default theory whose pseudo extension is neither an extension nor an inconsistent set. An example follows.

Let $\Delta=(D, W)$ be a default theory with

$$
\begin{aligned}
& D=\left\{\frac{: \mathbf{M} A}{B}, \frac{: \mathbf{M} \neg B}{C}, \frac{B \wedge C: \mathbf{M} A}{A}\right\} \\
& W=\emptyset .
\end{aligned}
$$

Then $F=T h(\{A, B, C\})$ is a pseudo extension of $\Delta$, but neither an extension of $\Delta$ nor an inconsistent set.

From the discussions above we can conclude the following :

(1) Pseudo extensions can successively be constructed from a given default theory.

(2) Extensions are always pseudo extensions.

(3) In a closed normal default theory, every pseudo extension is an extension if it is not inconsistent.

(4) If $W$ in $\Delta=(D, W)$ is inconsistent then $\Delta$ has a unique pseudo extension, which is inconsistent and coincides with the extension. This result immediately follows from Corollaries 2, 3 and the definition. 


\section{Extension Revision by Model Inference}

Default reasoning is non-monotonic in the sense that an increase in facts does not always mean an increase in knowledge. Thus the extension is not a definite knowledge, but may need to be revised, because some defaults used in constructing it become inapplicable when new facts are found. Reiter has defined the belief as a subset of the extension and has discussed revising it. In this section we define the belief as the whole set of extensions, and the belief revision as constructing an extension as similar as possible to the world to be described by the default theory. Thus we identify belief revision with extension revision. Let us consider the world for the theory to describe as an unknown model in a first order language. Then we can formalize the problem of extension revision in the framework of Shapiro's model inference $[3,4]$. Hereafter we assume readers are familiar with the model inference. Our problem can be expressed as follows:

Given a first order language $L$, an observational language

$$
L_{\circ}=\{\text { ground unit clauses of } L\}
$$

a hypothesis language $L_{h}=L$, and an oracle for an $h$-easy unknown model $K$ which satisfies the closed default theory $\Delta=(D, W)$, to find a finite $L_{0^{-}}$ complete axiomatization of $K$.

We say that the model $K$ satisfies a closed default theory $\Delta=(D, W)$ if it satisfies the following three conditions:

(1) $K \models W$

which means $K$ satisfies $W$.

(2) For a default

$$
\frac{\alpha: \mathbf{M} \beta_{1}, \ldots, \mathbf{M} \beta_{m}}{\omega} \in D
$$

if $K \models \alpha$ and $K \not \models \neg \beta_{1}, \ldots, K \not \forall \neg \beta_{m}$ then $K \mid=\omega$.

(3) Let $D^{\prime}$ be a subset of $D$ such that $W \cup \operatorname{Cons}\left(D^{\prime}\right)$ is consistent. Then

$$
W \cup \operatorname{Cons}\left(D^{\prime}\right) \forall u \Longrightarrow M \not u
$$

for any clause $u$ which is not a ground unit. 
The condition above restricts the unknown model $K$ in Shapiro's model inference.

A closed default theory $\Delta=(D, W)$ with finite $D$ and $W$ is called a finite closed default theory. For such a theory the following theorem holds :

Theorem 7 Let $\Delta=(D, W)$ be a finite closed default theory and $E$ be an extension of $\Delta$. Then there exists a finite $L_{o}$-complete axiomatization of $E$.

Proof It suffices to show $E \models T$ and $T \models L_{o}^{E}$ for some finite subset $T$ of $L$. By Theorem 1 any extension $E$ can be written as $E=\bigcup_{i=0}^{\infty} E_{i}$.

Let $D_{i}(i \geq 1)$ be the set of defaults which are used in defining $E_{i}$. Then $D_{i} \subseteq D$ and

$$
\begin{aligned}
& E_{0}=W \\
& E_{i}=T h\left(E_{i-1}\right) \cup \operatorname{Cons}\left(D_{i}\right) \quad(i \geq 1) .
\end{aligned}
$$

Since $D$ is finite, there exists an $N$ such that $D_{n}=D_{n-1}$ for any $n>N$. Hence we have

$$
E_{0} \subseteq E_{1} \subseteq \ldots \subseteq E_{N-1} \subseteq E_{N}=E_{N+1}=\ldots
$$

and

$$
E=\bigcup_{i=0}^{\infty} E_{i}=E_{N}
$$

We also have

$$
\begin{aligned}
E_{N} & =T h\left(E_{N-1}\right) \\
& =\operatorname{Th}\left(T h\left(E_{N-2}\right) \cup \operatorname{Cons}\left(D_{N-1}\right)\right) \\
& =\operatorname{Th}\left(T h\left(T h\left(E_{N-3}\right) \cup \operatorname{Cons}\left(D_{N-2}\right)\right) \cup \operatorname{Cons}\left(D_{N-1}\right)\right) \\
& =T h\left(T h\left(E_{N-3}\right) \cup \operatorname{Cons}\left(D_{N-2}\right) \cup \operatorname{Cons}\left(D_{N-1}\right)\right) \\
& \quad \vdots \\
& T h\left(T h\left(T h\left(E_{0}\right) \cup \operatorname{Cons}\left(D_{1}\right)\right) \cup \bigcup_{i=2}^{N-1} \operatorname{Cons}\left(D_{i}\right)\right) \\
& =T h\left(T h(W) \cup \bigcup_{i=1}^{N-1} \operatorname{Cons}\left(D_{i}\right)\right) \\
& =T h\left(W \cup \bigcup_{i=1}^{N-1} \operatorname{Cons}\left(D_{i}\right)\right) .
\end{aligned}
$$

Then

$$
T=W \cup \bigcup_{i=1}^{N-1} \operatorname{Cons}\left(D_{i}\right)
$$

is finite because so are $W, D$, and obviously $E \models T$ and $T \vdash L_{o}^{E}$. Therefore there exists a finite $L_{o}$-complete axiomatization of $E$. 
Theorem 8 Let $K$ be an $h$-easy unknown model which satisfies a finite closed default theory $\Delta=(D, W)$. If Shapiro's enumerative model inference algorithm identifies $K$ in the limit and produces a guess $T$ as the output, then $T h(T)$ is an extension of a default theory $\Delta^{\prime}=\left(D, W \cup W^{\prime}\right)$, where $W^{\prime}$ is a set of clauses.

\section{Proof Put}

$$
W^{\prime}=\left\{u \mid T \vdash u, W \cup \operatorname{Cons}\left(D^{\prime}\right) \forall u\right\} \cup\left\{f_{i} \mid 1 \leq i \leq k\right\},
$$

where $u$ is a ground unit clause and $f_{i}$ is defined by

$$
f_{i}= \begin{cases}\alpha_{i} & \text { if } V_{i}=\text { true } \\ \neg \alpha_{i} & \text { if } V_{i}=\text { false }\end{cases}
$$

when a fact $\left\langle\alpha_{i}, V_{i}\right\rangle$, a pair of ground atom and its truth value, is given as input to the algorithm.

In order to show that $T h(T)$ is an extension of $\Delta^{\prime}=\left(D, W \cup W^{\prime}\right)$, it suffices to show $T h(T)=\Gamma(T h(T))$. From the definition of $W^{\prime}$ and $K \models W$, obviously $T \vdash W \cup W^{\prime}$. Hence we have

$$
\begin{aligned}
& W \cup W^{\prime} \subseteq T h(T) \\
& T h(T h(T))=T h(T) .
\end{aligned}
$$

Since $K$ satisfies $\Delta$, there exists a default

$$
\delta=\frac{\alpha: \mathbf{M} \beta_{1}, \ldots, \mathbf{M} \beta_{m}}{\omega} \in D
$$

such that $K \models \alpha$ and $K \not \models \neg \beta_{1}, \ldots, K \not \models \neg \beta_{b}$ imply $K \models \omega$. Namely for a $\delta \in D$ if $T h(T) \vdash \alpha$ and $T h(T) \nvdash \neg \beta_{1}, \ldots, T h(T) \nvdash \neg \beta_{m}$ then $\omega \in T h(T)$. Hence $T h(T)$ satisfies the three conditions on $\Gamma(S)$. Therefore from the minimality of $\Gamma(S)$, we have

$$
\Gamma(T h(T)) \subseteq T h(T)
$$

We now show the converse. Let $v \in T h(T)$. There are four cases to be considered.

Case 1. $v \in W \cup W^{\prime}: v \in \Gamma(T h(T))$ by $W \cup W^{\prime} \subseteq \Gamma(T h(T))$.

Case 2. A default

$$
\delta=\frac{\alpha: \mathbf{M} \beta_{1}, \ldots, \mathbf{M} \beta_{m}}{v} \in D
$$


exists and $K \models \alpha, K \not \models \neg \beta, \ldots, K \not \models \beta_{m}$ : Clearly

$$
\begin{aligned}
& T h(T) \vdash \alpha \\
& T h(T) \forall \neg \beta_{1}, \ldots, T h(T) \forall \neg \beta_{m} \\
& T h(T) \subseteq \Gamma(T h(T)) .
\end{aligned}
$$

Hence $\Gamma(T h(T)) \vdash \alpha$ and $v \in \Gamma(T h(T))$.

Case 3. $W \cup W^{\prime} \cup \operatorname{Cons}\left(D^{\prime \prime}\right) \vdash v$ for the set $D^{\prime \prime}$ of all defaults

$$
\delta=\frac{\alpha: \mathbf{M} \beta_{1}, \ldots, \mathbf{M} \beta_{m}}{\omega} \in D
$$

such that $K \models \alpha, K \forall \neg \beta_{1}, \ldots, K \not \models \neg \beta_{m}$ : We have

$$
\begin{aligned}
v & \in T h\left(W \cup W^{\prime} \cup \operatorname{Cons}\left(D^{\prime \prime}\right)\right) \\
& \subseteq T h(\Gamma(T h(T))) \\
& =\Gamma(T h(T)) .
\end{aligned}
$$

Case 4. $\quad v \in T h(T)$ satisfies none of (1), (2), (3): If $v$ is not a ground unit then $K \not \forall v$ since $K$ satisfies $\Delta$. This contradicts that $L_{o}$ has an evidence to show the falsity of $T$. If $v$ is a ground unit then obviously

$$
v \in W^{\prime} \subseteq \Gamma(T h(T))
$$

Theorem 7 asserts that an $L_{o}$-complete axiomatization exists for any extension of finite closed default theory. Hence the extension appears in the enumeration $T h\left(T_{1}\right), T h\left(T_{2}\right), \ldots$, where $T_{1}, T_{2}, \ldots$ is the enumeration of all subsets of $L_{h}$. On the other hand Theorem 8 asserts that if we restrict the objects of the inference to the unknown models which are $h$-easy and satisfy finite closed default theories, then the models identified by the enumerative model inference algorithm are extensions of some closed default theories. The $\Delta^{\prime}$ in Theorem 8 is finite, and hence it has at most finite number of extensions. Therefore if for a finite closed default theory there exist two or more extensions in which the unknown model $K$ is included, the algorithm will find it in the search space of all extensions. Moreover if all the pseudo extensions of a finite closed default theory can be enumerated, the algorithm will find an extension for the unknown model by deleting guesses which are pseudo extensions but not extensions or contradict newly given facts. 


\section{Discussions}

There are some problems in Reiter's default theories. The extensions can not be constructed successively and even their existence is not guaranteed with exception of the closed normal cases. Many efforts to attack the problems have been made by many researchers. Reiter and Criscuolo [5] have introduced seminormal defaults. The seminormal default theories can describe some natural examples which the normal default theories can not, but still the existence of extensions is not guaranteed. Lukaszewicz [6] has studied a default theory of propositional logic and introduced another definition of extensions, for which the existence is guaranteed. Murakami et al. [7] have constructed a set of knowledge called a modified extensions, which is, in a sense, a generalization of Reiter's extension, and guaranteed the existence. [6,7] have thus guaranteed the existence for any default theory, but still their extensions can not be constructed successively from $W$. Murakami et al. [8] have dealt with this problem independently. They have defined sets of knowledge called a nonrecursive extensions for closed normal default theories. The nonrecursive extension can be uniquely determined.

In case the closed normal default theory $\Delta=(D, W)$ has an inconsistent extension, that is, $W$ is inconsistent, the nonrecursive extension is also inconsistent and equal to the extension of $\Delta$. However, the inconsistency of the nonrecursive extension does not mean that of $W$. In other words, if $\Delta$ has two or more extensions, the nonrecursive extension is inconsistent or equal to some of the extensions. If $\Delta$ has just one extension, the nonrecursive extension coincides with it.

On the other hand, our pseudo extensions may include non-extensions but include all the extensions. The extensions should be candidates for possible worlds. In this regard, our approach is better than the others.

We have introduced the notion of pseudo extensions which can be constructed successively and made a step towards the computerization of default reasoning. However, it is still not computable, because the condition $E_{i} \not \neg \neg \beta_{1}, \ldots, E_{i} \not \forall \beta_{m}$ for applying defaults is not even semi-computable. This aspect is common to all other approaches. To solve the problem we need some concept like the $h$-easiness. 


\section{References}

[1] Reiter, R. : A Logic for Default Reasoning, Artificial Intelligence, 13, 41-72 (1980).

[2] Shinmura, T. : On the Inconsistency in Non-monotonic Logic, Master's Thesis, Dept. of Information Systems, Kyushu Univ. (1986) (in Japanese).

[3] Shapiro, E.Y. : Inductive Inference of Theories From Facts, TR-192, Dept. Compt. Sci., Yale Univ. (1981).

[4] Ishizaka, H. : Model Inference Incorporating Least Generalization, Master's Thesis, Dept. Information Systems, Kyushu Univ. (1986) (in Japanese). To appear in J. Inf. Processing Soc. Japan.

[5] Reiter, R., Criscuolo, G. : On Interacting Defaults, Proc. 8th IJCAI, 270-276 (1983).

[6] Lukaszewicz, W. : Considerations on Default Logic, Proc. AAAI Non-monotonic Reasoning Workshop, 165-193 (1984).

[7] Murakami, K., Aibara, T., Shitanda, H. : Modified Extension and Its Properties in Default Reasoning, Trans. Inf. Processing Soc. Japan, 12, 1280-1287 (1987) (in Japanese).

[8] Murakami, K. Aibara, T., Shitanda, H. : Nonrecursive Extension and Its Properties in Default Reasoning, J. Japanese Soc. Artificial. Intelligence, 3, 359-367 (1988) (in Japanese).

[9] Yuasa, H.: Pseudo Extensions in Default Reasoning and Belief Revision Using Model Inference, Master's Thesis, Dept. Information Systems, Kyushu Univ. (1988) (in Japanese). 